\title{
Precision and accuracy in film stiffness measurement by Brillouin spectroscopy
}

\author{
M. G. Beghi, ${ }^{1}$ F. Di Fonzo, ${ }^{2}$ S. Pietralunga,${ }^{3}$ C. Ubaldi, ${ }^{3}$ and C. E. Bottani ${ }^{1}$ \\ ${ }^{1}$ Energy Department and NEMAS Center, Politecnico di Milano, Via Ponzio 34/3, 20133 Milano, Italy \\ ${ }^{2}$ Center for NanoScience and Technology of IIT@ PoliMi, Via Pascoli 70/3, 20133 Milano, Italy \\ ${ }^{3}$ Fondazione Politecnico di Milano-PoliCom, Via G. Colombo 81, 20133 Milano, Italy
}

(Received 22 December 2010; accepted 8 April 2011; published online 27 May 2011)

\begin{abstract}
The interest in the measurement of the elastic properties of thin films is witnessed by a number of new techniques being proposed. However, the precision of results is seldom assessed in detail. Brillouin spectroscopy (BS) is an established optical, contactless, non-destructive technique, which provides a full elastic characterization of bulk materials and thin films. In the present work, the whole process of measurement of the elastic moduli by BS is critically analyzed: experimental setup, data recording, calibration, and calculation of the elastic moduli. It is shown that combining BS with ellipsometry a fully optical characterization can be obtained. The key factors affecting uncertainty of the results are identified and discussed. A procedure is proposed to discriminate factors affecting the precision from those affecting the accuracy. By the characterization of a model transparent material, silica in bulk and film form, it is demonstrated that both precision and accuracy of the elastic moduli measured by BS can reach 1\% range, qualifying BS as a reference technique. (ㅇ 2011 American Institute of Physics. [doi:10.1063/1.3585980]
\end{abstract}

\section{INTRODUCTION}

The stiffness of films depends on the film microstructure and its precise characterization is crucial when thin layers have structural functions, as in micro-electro-mechanical systems. The interest in its measurement is witnessed by the number of new techniques being proposed. In the most widespread method to measure the mechanical properties of films, nano-indentation, both elastic and inelastic deformations occur, and are characterized. Nevertheless, the information must be disentangled from a complex strain history, and a single parameter, the reduced modulus, is obtained to represent stiffness. Furthermore, in the indentation of supported films the effects of substrate and tip properties have to be avoided or deconvoluted. ${ }^{2}$

The interest in a direct and complete elastic characterization leads to measurement methods involving only elastic strains; they exploit either propagating acoustic waves or standing oscillations, excitation being monochromatic (e.g., resonance techniques ${ }^{3-5}$ ) or broadband, with spectral analysis of the response (laser acoustic techniques, where excitation is impulsive. $\left.{ }^{6-10}\right)$. The direct outcome is the propagation velocity of the vibrational modes; the latter being determined by stiffness and inertia, the elastic moduli are obtained if the mass density is known.

Recently proposed methods exploit relatively sophisticated optical techniques such as pump and probe with femtosecond laser pulses $\mathrm{s}^{7-10}$ or laser-Doppler interferometry ${ }^{5}$ and can require specimen manipulations such as the deposition of a metallic layer ${ }^{7-10}$ or a pattern definition by microlithography techniques..$^{10}$ The so-called picosecond ultrasonics ${ }^{7-10}$ is an evolution of the laser ultrasonics technique. ${ }^{6}$ Brillouin spectroscopy (BS) (Refs. 11 and 12) and surface Brillouin spectroscopy (SBS) are well known and relatively simpler optical techniques which measure ultrasonic excitations at sub-micrometric wavelengths. Without requiring sample manipulations, they only need surfaces of reasonable quality and are applicable down to sub-millimetric sample sizes. Having been exploited for a long time for the measurement of the elastic properties of bulk materials ${ }^{12-14}$ and of films, ${ }^{12,15-17}$ they are finding new applications. ${ }^{18-27}$

The accuracy of the elastic characterization achievable by SBS was somehow puzzling since the pioneering work of Sandercock. ${ }^{28}$ Although significant insight has been gained on the precision of spectra, ${ }^{13,29}$ a comprehensive assessment of the whole measurement process, down to the final results in terms of elastic moduli, does not seem to have been performed. Such an assessment is the objective of this work. The usefulness of combining BS and SBS with ellipsometry, obtaining a fully optical technique, naturally emerges. A data analysis procedure is obtained, which evaluates the uncertainties, discriminating precision from accuracy. This procedure is tested on a model system, a silica film, in comparison with bulk silica; it is found that the precision achievable by this optical technique can exceed that of other methods.

\section{INELASTIC LIGHT SCATTERING BY BULK AND SURFACE ELASTIC WAVES}

The elastic continuum model characterizes a medium by the mass density $\rho$ and the matrix of the elastic constants $C_{i j}$, which, in the isotropic case, is fully determined by any two quantities among $C_{11}, C_{44}$ (which coincides with the shear modulus), Young's modulus $E$, Poisson's ratio $v$, and bulk modulus $B$. A homogeneous isotropic continuum supports elastic waves, both bulk longitudinal (BL) and bulk transversal (BT) waves, whose velocities are, 


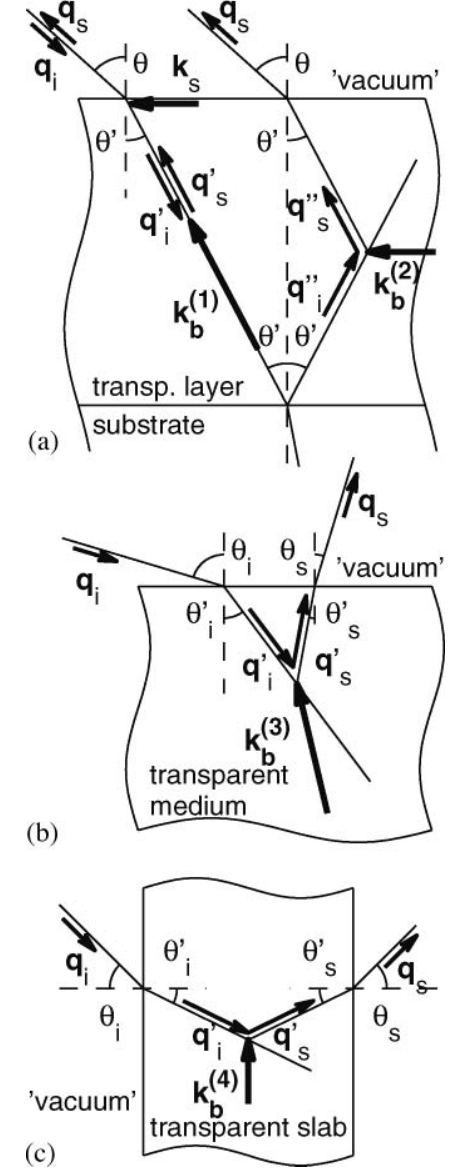

FIG. 1. The main Brillouin scattering geometries (thin arrows: optical wavevectors; thick arrows: acoustic wavevectors). (a) backscattering, at incidence angle $\theta$, direct backscattering by a surface acoustic wave of wavevector $\mathbf{k}_{\mathrm{s}}$ or a bulk acoustic wave of wavevector $\mathbf{k}_{\mathrm{b}}{ }^{(1)}$; for a transparent film on a reflecting substrate, indirect backscattering by a bulk acoustic wave of wavevector $\mathbf{k}_{\mathbf{b}}{ }^{(2)}$. (b) Forward scattering, with incidence angle $\theta_{i}$ and light collection at angle $\theta_{s}$ and (c) transmission scattering, with incidence angle $\theta_{i}$ and light collection at angle $\theta_{s}$.

respectively, ${ }^{30}$

$$
v_{l}=\sqrt{\frac{C_{11}}{\rho}}, \quad v_{t}=\sqrt{\frac{C_{44}}{\rho}},
$$

and, at a free surface, the Rayleigh wave, the paradigm of surface waves, having velocity $v_{R}$. The basic relations among the moduli and the velocities are summarized in Part A of the Supplementary material. ${ }^{1}$

In BS and SBS, the sample is illuminated by a laser beam and the spectrum of scattered light is measured. The spectrum contains the Stokes/anti-Stokes doublets due to inelastic scattering by thermally excited vibrations, which are probed at the wavevector selected by the scattering geometry; the main configurations are summarized in Fig. 1. The incident beam, of angular frequency $\Omega_{\mathrm{i}}$ and wavelength $\lambda_{0}$, impinging on the sample with wavevector $\mathbf{q}_{i}$, is refracted (in sufficiently transparent materials) into wavevector $\mathbf{q}_{i}^{\prime}$. Scattered light, of wavevector $\mathbf{q}_{s}^{\prime}$, emerges with wavevector $\mathbf{q}_{s}$. The probed wavevector, $\mathbf{k}= \pm\left(\mathbf{q}_{s}^{\prime}-\mathbf{q}_{i}^{\prime}\right)$, is determined by $\lambda_{0}$, the directions of $\mathbf{q}_{i}$ and $\mathbf{q}_{s}$, and, possibly, the refractive index $n$. Light inelastically scattered by a vibrational excitation of angular frequency $\omega(\mathbf{k})$ has frequency $\Omega_{\mathrm{s}}$ and allows to measure $\omega=\left|\Omega_{\mathrm{s}}-\Omega_{\mathrm{i}}\right|$ and the excitation velocity $v=\omega / k$; a precise knowledge of $|\mathbf{k}|=k$ is therefore crucial. The wavevectors $\mathbf{k}$ for the geometries of Fig. 1 are discussed in Part B of the Supplementary material; ${ }^{1}$ the "geometrical wavelength" $\lambda^{\prime}$ can be introduced, which only depends on $\lambda_{0}$ and on geometrical factors (Eqs. (B.1)-(B.5)). It is the acoustic wavelength that would be probed if $n$ was unity such that

$$
k=\left(\frac{2 \pi}{\lambda^{\prime}}\right) n
$$

The actually probed wavelength $2 \pi / k$ coincides with $\lambda^{\prime}$ when $\mathbf{k}$ does not depend on $n$ (Eqs. (B.2) and (B.5)), otherwise it is $\lambda^{\prime} / n$, either exactly (Eq. (B.1)) or approximately (Eqs. (B.3) and (B.4)).

Spectral doublets are expected for each probed wavevector, at frequency shifts

$$
f=\frac{\omega}{2 \pi}=\frac{\left|\Omega_{s}-\Omega_{i}\right|}{2 \pi}=\frac{v k}{2 \pi}=\frac{v}{\lambda^{\prime}} n,
$$

one doublet for surface waves, where $k$ stands for $\left|\mathbf{k}_{\mathrm{s}}\right|$ and $v$ for $v_{R}$, and two doublets for bulk waves, where $k$ stands for $\left|\mathbf{k}_{\mathrm{b}}\right|$ and $v$ stands, respectively, for $v_{l}$ and $v_{t}$, for the BL and BT waves. Only in direct backscattering $\left(\mathbf{q}_{s}^{\prime}=-\mathbf{q}_{i}^{\prime}\right)$ the scattering cross section for the BT wave is null, and the corresponding doublet is not expected, ${ }^{13}$ otherwise both doublets can be measured.

\section{EXPERIMENTS}

The present investigation is performed on two types of amorphous silica $\left(\mathrm{SiO}_{2}\right)$ : a fused silica platelet, several millimeters wide and $\sim 2 \mathrm{~mm}$ thick; supplied by a producer of indentation instrumentation (Micro Materials Ltd.) as a reference sample for the indenter calibration, with nominal values $E=72 \mathrm{GPa}$ and $v=0.18$ and a silica film, slightly thicker than $2 \mu \mathrm{m}$, thermally grown at the surface of a $\mathrm{Si}(001)$ wafer, in a step of an industrial process. ${ }^{31}$

The experimental setup of Fig. 2 realizes all the geometries of Fig. 1. If the beam splitter $\mathrm{BeS}$ is removed, the external scattering angle is $90^{\circ}$, in either the forward scattering or the transmission configuration, with any value of incidence angle. If the mirror M1 replaces BeS, backscattering is achieved alone. When both BeS and M1 are in place, the spectrum

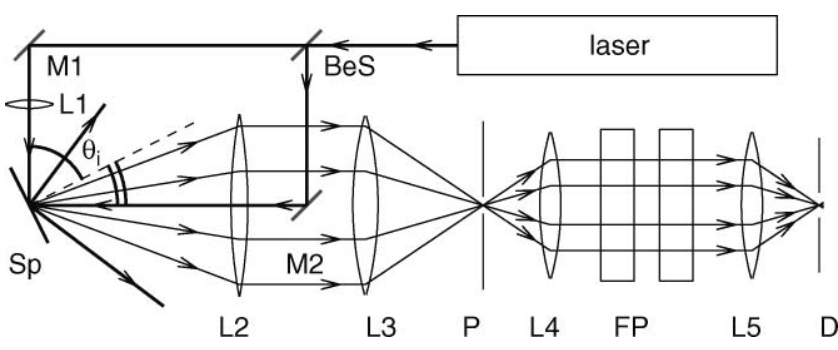

FIG. 2. Experimental setup for Brillouin spectroscopy. BeS: beam splitter; M1, M2: mirrors; Sp: sample; L1-L5: lenses; P: entrance pinhole of the spectrometer; FP: Fabry-Perot interferometer; D: light detector. With BeS in place the backscattering and the $90^{\circ}$ scattering geometries are superposed; $90^{\circ}$ scattering alone is obtained removing BeS, backscattering alone is obtained substituting BeS by M1. Additional details, such as the mirrors which steer the scattered beam, are not shown. 
contains the doublets due to both geometries, allowing more precise measurements of the ratios of doublet frequencies. Typically, in this configuration BeS reflects only a weak "witness beam," useful to achieve the required coincidence of the focal points of the focusing and the light collecting lenses (L1 and L2 of Fig. 2). In the symmetric forward scattering geometry (Fig. 1(b)), the mirror which steers the witness beam also suppresses, as needed, the specularly reflected beam.

The light source is an $\mathrm{Ar}^{+}$laser (Innova 300, Coherent Inc.), operating at power of $200 \mathrm{~mW}$ and wavelength $\lambda_{0}$ $=514.5 \mathrm{~nm}$. The incident beam is polarized either parallel or orthogonal to the plane of incidence. Light is collected without polarization analysis, except for tests of peak attributions; the incident polarization is preserved in scattering by BL and Rayleigh waves and rotated in scattering by BT waves. ${ }^{13}$ The spectra of the scattered light are analyzed by a $3+3$ pass tandem Fabry-Perot interferometer of the Sandercock type. ${ }^{32-34}$

Measurements are performed, for the bulk sample, in all the geometries of Fig. 1. For the silica film, the geometry of Fig. 1(c) is precluded; that of Fig. 1(b) is viable, but did not give clear enough spectral doublets. Acoustic modes with wavevector normal to the surface were indeed measured in micrometric films, but from polymer films, ${ }^{22}$ which typically have large scattering cross sections or in direct backscattering with normal incidence. ${ }^{35}$ Backscattering from a film probes the acoustic wavevectors $\mathbf{k}_{\mathrm{s}}, \mathbf{k}_{\mathrm{b}}{ }^{(2)}$, and $\mathbf{k}_{\mathrm{b}}{ }^{(1)}$. The first two coincide, do not depend on $n$, are parallel to the surface and correspond to wavelengths $\lambda^{\prime}=\lambda_{0} /(2 \sin \theta$ ) (see Eq. (A.6)) which, for $\theta \geq 30^{\circ}$, do not exceed $0.51 \mu \mathrm{m}$; the latter correspond to wavelength $\lambda^{\prime} / n=\lambda_{0} /(2 n)$ (see Eq. (A.5)) which, with $n$ above 1.45 , does not exceed $0.18 \mu \mathrm{m}$. Since the layer thickness exceeds $2 \mu \mathrm{m}$, the vibrational excitations, it supports, at these wavelengths are indistinguishable from those in a semi-infinite solid.

If a same acoustic mode is observed in different geometries, the ratio of the frequency shifts coincides with the ratio of the wavevector magnitudes, which in turn depends on the scattering geometries and the refractive index (Eqs. (A.5)-(A.9)). From these ratios of frequency shifts, the refractive index can be derived. The resulting values are in agreement with those obtained by ellipsometry, but more scattered, mainly because of the relatively weak dependence of the modulus $k$ on $n$. In order to obtain the best precision and accuracy, the values of the refractive index obtained by variable angle spectroscopic ellipsometry (VASE) (Ref. 36) are adopted.

Measurements are performed by a WVASE32 ellipsometer (Woollam Co., Inc.), with incidence angles of $65^{\circ}, 70^{\circ}$, and $75^{\circ}$, and wavelength spanning the $500-1600 \mathrm{~nm}$ interval. The raw results, the polarization resolved complex coefficients of reflection, are analyzed by a fitting procedure which crucially depends on the choice of the model for the sample itself. The model includes the structure of the sample and its optical constants, by either their initial values or the functional form of their dispersion. The accuracy of the measurement is ruled by the precision in the alignment of the polarizing components, while the accuracy for the parameter derivation depends on the choice of the model. In case of hardware operating at best nominal performance, the achievable uncertainty is estimated in the range of $\sigma_{n}=(2 \div 3) \times 10^{-3} .37,38$

The two silica samples are modeled by, respectively, homogeneous amorphous silica, and a single layer of homogeneous amorphous silica over a $\mathrm{Si}$ substrate. The substrate is modeled by tabulated values for the refractive index, ${ }^{39}$ and the initial guess for silica includes the $n(\lambda)$ values from Ref. 40, and a null absorption. The fitting procedure is performed on the silica optical constants and, for the film, the silica thickness. In the latter case the results are consistent with a homogeneous and amorphous dielectric film, $2292.4 \mathrm{~nm}$ thick, of negligible surface roughness. The level of depolarization on the signal is compatible with a $1.83 \%$ degree of thickness non-uniformity. The resulting values of refractive index at the wavelength $\lambda_{0}=514.5 \mathrm{~nm}$ are, respectively, $n_{b}=1.467$ for the bulk silica, which is in good agreement with Ref. 39, and, for the film, $n_{f}=1.488$, which is measurably higher than that of other forms of silica. In both cases, due to the simplicity of the measured systems, the uncertainties can be estimated in the lower band achievable by ellipsometry: $\sigma_{n} \approx 0.003$, meaning $\sigma_{n} / n \approx 2 \times 10^{-3}$.

The different refractive indexes indicate different mass densities. The Lorentz-Lorenz relation ${ }^{41}$ states that, for molecules of given polarizability, the number of molecules per unit volume is proportional to $\left(n^{2}-1\right) /\left(n^{2}+2\right)$. The values $n_{b}$ and $n_{f}$, therefore, allow to estimate the film mass density $\rho_{f}$ and its uncertainty $\sigma_{\rho_{f}}$ from the bulk values $\rho_{b}$ and $\sigma_{\rho_{b}}$ (see Part D of the Supplementary material ${ }^{1}$ ). For bulk silica the value $\rho_{b}=2200 \mathrm{~kg} / \mathrm{m}^{3}$ is widely accepted, whose uncertainty is not easily assessed; an estimate of $\sigma_{\rho, b} \approx 5 \mathrm{~kg} / \mathrm{m}^{3}$, meaning $\sigma_{\rho, b} / \rho_{b} \approx 0.23 \%$, seems reasonable.

With the above values, Eqs. (D.1) and (D.3) give $\rho_{f}=2284 \mathrm{~kg} / \mathrm{m}^{3}$, with $\sigma_{\rho, f} / \rho_{\mathrm{f}} \approx 8 \times 10^{-3}$, i.e., $\sigma_{\rho, f}$ $\approx 18 \mathrm{~kg} / \mathrm{m}^{3}$. The ranges of variability can also be assessed in a more "deterministic" way, considering all the variations $\mathrm{d} \rho_{b}= \pm \sigma_{\rho, b}, \mathrm{~d} n_{b}= \pm \sigma_{n_{b}}, \mathrm{~d} n_{f}= \pm \sigma_{n_{f}}$. and computing $\rho_{f}$ from all the possible combinations. Equation (D.2) gives for $\rho_{f}$ values in the range $2284 \pm 30 \mathrm{~kg} / \mathrm{m}^{3}$. However, a simultaneous significant underestimation of $n_{b}$ and overestimation of $n_{f}$ (or the reverse) is deemed unlikely. A more credible range of variation is obtained considering only the variations of $n_{f}$ and $\rho_{b}$. Equation (D.2) shows that the largest variations are found for $\mathrm{d} \rho_{b}$ and $\mathrm{d} n_{f}$ of the same sign: $\left(\mathrm{d} \rho_{\mathrm{b}} / \rho_{\mathrm{b}}, \mathrm{d} n_{\mathrm{f}} / n_{\mathrm{f}}\right)$ $= \pm(0.23 \%, 0.2 \%)$, giving $\mathrm{d} \rho_{\mathrm{f}} / \rho_{\mathrm{f}}= \pm 0.75 \%$, i.e., $\rho_{\mathrm{f}}=2284$ $\pm 18 \mathrm{~kg} / \mathrm{m}^{3}$.

The Fabry-Perot interferometer, operated as a tunable band-pass filter (see Part C of the Supplementary material ${ }^{1}$ ), supplies the raw spectrum as photon count number vs. channel number. A common procedure to perform frequency calibration exploits the spectral "ghosts;" more sophisticated calibration schemes, by reference spectral lines, have been exploited, ${ }^{42}$ but seem justified only by special requirements. The, generally non-integer, "channel numbers" $n_{p}{ }^{+}$and $n_{p}{ }^{-}$ of the Stokes/anti-Stokes peaks of a spectral doublet are identified by a best fit procedure, as well as the "channel numbers" $n_{\mathrm{g}}{ }^{+}$and $n_{\mathrm{g}}{ }^{-}$of the spectral "ghosts" (see Part C of the Supplementary material). The frequency shifts $\pm F S R$ being attributed to the "channel numbers" $n_{\mathrm{g}}{ }^{+}$and $n_{\mathrm{g}}{ }^{-}$, the frequency 


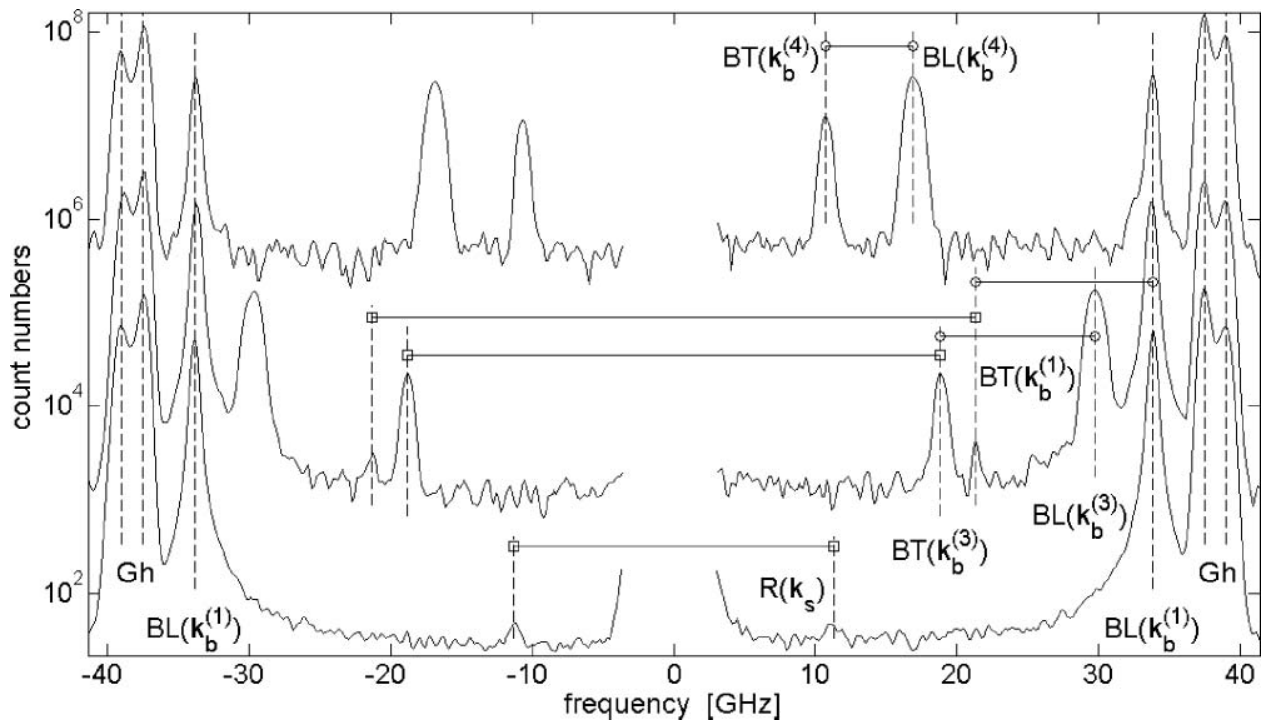

FIG. 3. Brillouin spectra from the bulk silica sample. Bottom to top (see also Fig. 2): backscattering alone; $90^{\circ}$ forward scattering (Fig. 1(b)) together with backscattering; $90^{\circ}$ transmission scattering (Fig. 1(c)) together with backscattering. Incident light polarized normal to the incidence plane. For clarity, the second and third spectra are shifted by two and four decades, and the strong peak at null frequency shift, due to elastically scattered light, is removed. Peak labels: Gh: instrumental "ghosts" due to elastically scattered light; R: Rayleigh wave of probed wavevector $\mathbf{k}_{\mathrm{s}}$; BL and BT: bulk longitudinal and bulk transversal waves, of probed wavevectors $\mathbf{k}_{\mathbf{b}}{ }^{(1)}, \mathbf{k}_{\mathbf{b}}{ }^{(3)}$, and $\mathbf{k}_{\mathbf{b}}{ }^{(4)}$ (see Fig. 1). Lines with square marker connect some Stokes/anti-Stokes doublets; lines with a circle marker connect the peaks due to the BL and BT waves probed by the same wavevector.

shift $f$ of a doublet is

$$
f=F S R \frac{n_{p}^{+}-n_{p}^{-}}{n_{g}^{+}-n_{g}^{-}} .
$$

Spectra obtained from the bulk sample are shown in Fig. 3. The mirror spacing is $4 \mathrm{~mm}$, giving $F S R \approx 37.5$ $\mathrm{GHz}$. In all the spectra, an intense doublet is due to direct backscattering (wavevector $\mathbf{k}_{\mathrm{b}}{ }^{(1)}$ ), by the BL wave, of at least a "witness beam." If the main beam is in this geometry (lowest spectrum), the doublet due to the Rayleigh wave is also visible, otherwise (upper spectra) it is too weak. The peaks in the upper spectra, recorded in different $90^{\circ}$ scattering geometries, have a different shape, showing the different consequences of the same light collection angle, discussed in Part C of the Supplementary material. In these geometries, both doublets due to the BL and the BT waves are evident. This attribution is confirmed by the identical value of the $\mathrm{BL} / \mathrm{BT}$ frequency ratio, which also leads to assign the further weak doublet in the intermediate spectrum to backscattering by the BT wave. The cross section for this wave, null in direct backscattering, is not null in its neighborhood, giving some scattering, collected by the finite collection angle.

Spectra obtained from the transparent silica film are shown in Fig. 4. At higher frequencies, the doublets due to scattering by bulk waves of silicon become visible. The doublets observed in backscattering have lower intensity than those from the bulk sample due to the smaller depth of the scattering volume; the uncertainties in the identification of the "channel numbers" $n_{p}{ }^{+}$and $n_{p}{ }^{-}$are larger, those of $n_{g}{ }^{+}$ and $n_{g}{ }^{-}$remaining instead comparable. Direct backscattering (wavevector $\mathbf{k}_{\mathrm{b}}{ }^{(1)}$ ) only gives the doublet due to the $\mathrm{BL}$ wave, while indirect backscattering (wavevector $\mathbf{k}_{\mathbf{b}}{ }^{(2)}$ ) also gives the doublet due to the BT wave. The reflections at the surfaces depending on the polarization of light, the intensities of the doublets due to indirect backscattering and to scattering by the Rayleigh wave strongly depend on polarization, as shown by Fig. 4; the upper two spectra differ only for the switch of the incident polarization by a $\lambda / 2$ plate.

\section{ASSESSMENT OF THE UNCERTAINTIES}

From each measured frequency shift $f$ the acoustic velocity $v$ is given by (Eqs. (2) and (3))

$$
v=\frac{2 \pi f}{k}=\frac{\lambda^{\prime} f}{n}=\frac{v^{\prime}}{n},
$$

where, similarly to Eq. (2), the velocity $v^{\prime}=\lambda^{\prime} f$ is the value that would be obtained if the refractive index $n$ was unity, and the presence or not of the inverse proportionality on $n$ depends on the exchanged wavevector. The uncertainty $\sigma_{v}$ of velocity depends on the uncertainties $\sigma_{f}, \sigma_{k}, \sigma_{\lambda^{\prime}}$, and $\sigma_{n}$ of the respective quantities; from Eq. (5), the error propagation formula gives

$$
\begin{aligned}
\left(\frac{\sigma_{v}}{v}\right)^{2} & =\left(\frac{\sigma_{f}}{f}\right)^{2}+\left(\frac{\sigma_{k}}{k}\right)^{2}=\left(\frac{\sigma_{f}}{f}\right)^{2}+\left(\frac{\sigma_{\lambda^{\prime}}}{\lambda^{\prime}}\right)^{2}+\left(\frac{\sigma_{n}}{n}\right)^{2} \\
& =\left(\frac{\sigma_{v^{\prime}}}{v^{\prime}}\right)^{2}+\left(\frac{\sigma_{n}}{n}\right)^{2} .
\end{aligned}
$$

The contribution $\sigma_{k} / k$ is split into the terms $\sigma_{\lambda^{\prime}} / \lambda^{\prime}$ and $\sigma_{n} / n$, and the first of them is grouped with $\sigma_{f} / f$ to give $\sigma_{v}, / v^{\prime}$, following the different nature of the uncertainties. Both $\sigma_{f}$ and $\sigma_{\lambda^{\prime}}$ are due to intrinsically random errors, respectively, in the identification of the doublet frequency and in setting the scattering geometry, which tend to be averaged out by repeated measurements. They tend to scatter the results, without affecting their mean value. As well as $\sigma_{v^{\prime}}$, which groups them, they characterize a lack of precision, not of accuracy. Instead, the value of $n$, a parameter known to a finite accuracy, is identical in repeated measurements. Its uncertainty affects the mean value of results, not their scatter; thus affecting 


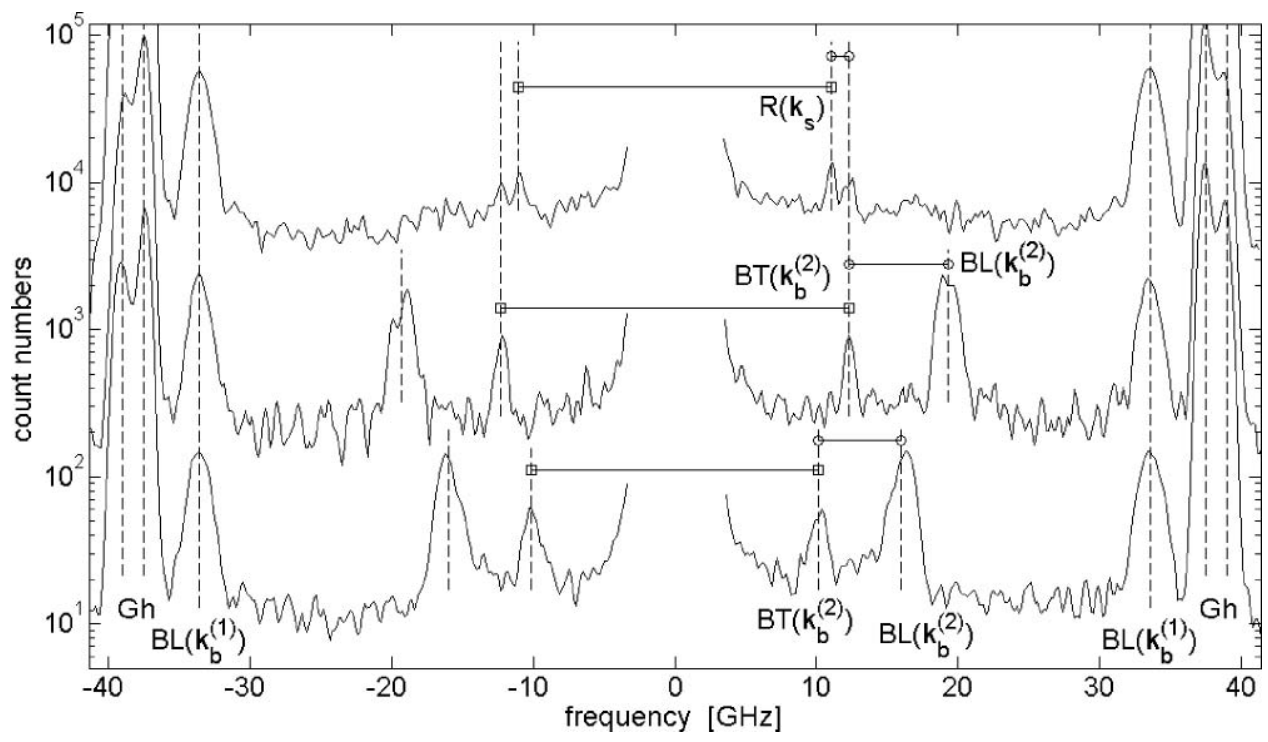

FIG. 4. Brillouin spectra measured in backscattering from the film silica sample. Bottom to top: $45^{\circ}$ and $60^{\circ}$ incidence angles, with incident light polarized normal to the incidence plane; $60^{\circ}$ incidence angle, with incident light polarized in the incidence plane. Scattered light collected without polarization analysis. For clarity, the second and third spectra are shifted, and the peak due to elastically scattered light is removed. Peak labels and markers: same of Fig. 3.

accuracy, but not precision. If the wavevector $k$ does not depend on $n, v$ coincides with $v^{\prime}$ and $\sigma_{v}$ with $\sigma_{v^{\prime}}$. For the exchanged wavevectors proportional to $n$ (see Eqs. (2) and (5)) $v=v^{\prime} / n$ and the further uncertainty $\sigma^{\prime}{ }_{v}=\sigma_{v^{\prime}} / n$ is usefully introduced, such that $\sigma^{\prime}{ }_{v} / v=\sigma_{v^{\prime}} / v^{\prime}$. The uncertainties are thus compared to the same velocity $v, \sigma^{\prime}{ }_{v}$ characterizes the scatter of results, while $\sigma_{v}$ also includes the possible inaccuracy due to $n$.

For $\sigma_{f} / f$, Eq. (4) gives

$$
\begin{aligned}
\left(\frac{\sigma_{f}}{f}\right)^{2} & =\left(\frac{\sigma_{F S R}}{F S R}\right)^{2}+\frac{\sigma_{n_{p}^{+}}^{2}+\sigma_{n_{p}^{-}}^{2}}{\left(n_{p}^{+}-n_{p}^{-}\right)^{2}}+\frac{\sigma_{n_{g}^{+}}^{2}+\sigma_{n_{g}^{-}}^{2}}{\left(n_{g}^{+}-n_{g}^{-}\right)^{2}} \\
& =\left(\frac{\sigma_{F S R}}{F S R}\right)^{2}+\left(\frac{\sigma_{p}}{n_{p}}\right)^{2}+\left(\frac{\sigma_{g}}{n_{g}}\right)^{2},
\end{aligned}
$$

where the last two terms are the relative uncertainties remaining from the best fit procedures. The first term on the rhs is due to the spacing $d$, inversely proportional to FSR. An optical calibration $^{34}$ can achieve a relative uncertainty $\sigma_{d} / d$ $=\sigma_{F S R} / F S R$ below a micrometer over a few millimeters: the value $\sigma_{d} / d=2 \times 10^{-4}$ is considered here, but it must be remembered that this value rises to $10^{-3}$ if a spacing below $1 \mathrm{~mm}$, i.e., FSR above $150 \mathrm{GHz}$, is needed. The term $\sigma_{p} / n_{p}$ in Eq. (7), due to the doublet, mainly depends on the signal-to-noise ratio. For strong peaks $\left(\mathrm{BL}\left(\mathbf{k}_{\mathrm{b}}{ }^{(1)}\right)\right.$ in Fig. 3), it turns out to be below $10^{-3}$, down to $10^{-4}$, while for the weakest ones $\left(\mathrm{R}\left(\mathbf{k}_{\mathrm{s}}\right)\right.$ in Figs. 3 and 4$)$ it can rise to $10^{-2}$. The term $\sigma_{g} / n_{g}$, due to the spectral ghosts, turns out to be always below $10^{-3}$, but remains above $10^{-4}$, despite the high intensity, due to the difficulty of adequately describing the peculiar shape of the double peaked ghosts (see Part C of the Supplementary material).

Despite this difficulty, the separate calibration of each spectrum is confirmed as best choice. The single "channel numbers" $n_{\mathrm{g}}{ }^{+}$and $n_{\mathrm{g}}{ }^{-}$from spectra recorded in nominally identical conditions turn out to differ by even more than one part in a thousand, probably due to either minor differences in the measurement setup or to thermal drifts. Therefore, a calibration performed, once in a while, against a sharp doublet, would give larger uncertainties.

For $\sigma_{\lambda^{\prime}} / \lambda^{\prime}$, in Part B of the Supplementary material it is shown that it is null for direct backscattering, in which the wavevector $\mathbf{k}_{\mathrm{b}}{ }^{(1)}$ is proportional to $n$ (Eq. (B.1)), while it is $\sigma_{\lambda^{\prime}} / \lambda^{\prime}=\sigma_{\theta} / \tan \theta$ in backscattering (Eq. (B.6)), for the exchanged wavevectors $\mathbf{k}_{\mathrm{b}}{ }^{(2)}=\mathbf{k}_{\mathrm{s}}$, parallel to the surface and independent from $n$, (Eq. (B.2)). The error $\sigma_{\theta}$ is mainly connected to the identification of a reference position like normal incidence. For $\sigma_{\theta} \approx 0.2^{\circ} \approx 3.5 \mathrm{mrad}$ (Ref. 29) the relative uncertainty $\sigma_{\lambda^{\prime}} / \lambda^{\prime}$ is up to $10^{-2}$ at $\theta=20^{\circ}$, decreasing to 1.3 $\times 10^{-3}$ at $\theta=70^{\circ}$. By optimizing the detection of misalignments, this uncertainty can be reduced below $\sigma_{\theta} \approx 1 \mathrm{mrad}$, leading to values of $\sigma_{\lambda^{\prime}} / \lambda^{\prime}$ still almost $3 \times 10^{-3}$ at $\theta=20^{\circ}$, but below $2 \times 10^{-3}$ at $\theta=30^{\circ}$ and below $4 \times 10^{-4}$ at $\theta=70^{\circ}$.

The assessment of $\sigma_{\lambda^{\prime}} / \lambda^{\prime}$ is more elaborate for the $90^{\circ}$ geometries (Eq. (B.3) and its homologous for the transmission geometry), in which the external scattering angle $\delta$, with its uncertainty $\sigma_{\delta}$, also has a role. As discussed in Part B of the Supplementary material, in our setup procedure only the uncertainties in $\theta_{i}$ and in $\delta$ are uncorrelated, and therefore allow to exploit the usual error propagation formula. Table B.I in Part B of the Supplementary material presents numerical evaluations for $\sigma_{\theta} \approx 1 \mathrm{mrad}$, mentioned above, and for a conservative estimation, in our present setup, of $\sigma_{\delta} \approx 4 \mathrm{mrad}$.

Ellipsometry gives, as noted above, $\sigma_{n} / n \approx 2 \times 10^{-3}$. The various uncertainties, including $\sigma_{\lambda^{\prime}} / \lambda^{\prime}$ of Table B.I., are summarized in Table I. In each of its lines at least one contribution is of the order of $10^{-3}$, showing that, although by different limiting factors, this is a lower bound for the relative uncertainty achievable in a single measurement of velocity by BS and SBS. Below that level, further contributions, neglected in Eqs. (B.3), (B.4), (B.7), (6), and (7), become non-negligible, non-perfect linearity between mirror spacing and channel number, ${ }^{34}$ non-linearity of the spacing vs. frequency relationship (exact linearity is between spacing and wavelength). 
TABLE I. For the velocities $v_{l}, v_{t}$, and $v_{R}$ measured in the various geometries, contributions to the relative uncertainties $\sigma_{v} / v$ due to the various causes discussed in the text. Null contributions are indicated by zero when they are null only in the specified conditions, and by a dash when they are intrinsically null. The values of $\sigma_{p} / n_{p}$ report the ranges of values found for the various cases. For $\sigma_{g} / n_{g}$ the range of observed value is $\approx(1 \div 8) \times 10^{-4}$, for all the cases. The reported typical value results from the combination (Eq. (7)) with $\sigma_{F S R} / F S R \approx 2 \times 10^{-4}$, which also is same for all the cases.

\begin{tabular}{|c|c|c|c|c|c|}
\hline \multirow[b]{2}{*}{ Velocity, geometry } & \multicolumn{5}{|c|}{ Primary uncertainty } \\
\hline & $\sigma_{p} / n_{p}$ & $\sigma_{g} / n_{g}$ & $\sigma_{\theta_{i}} \approx 1 \mathrm{mrad}$ & $\begin{array}{c}\sigma_{\delta} \approx 4 \mathrm{mrad} \\
\left(\delta=90^{\circ}, \theta_{i}=45^{\circ}\right)\end{array}$ & $\begin{array}{c}\sigma_{n} / n \approx 2 \times 10^{-3} \\
\left(\delta=90^{\circ}, \theta_{i}=45^{\circ}\right)\end{array}$ \\
\hline$v_{l}\left(\mathbf{k}_{\mathrm{b}}^{(1)}\right),($ bulk $)$ & $(1 \div 4) \times 10^{-4}$ & typ. $5 \times 10^{-4}$ & - & - & $2 \times 10^{-3}$ \\
\hline$v_{t}\left(\mathbf{k}_{\mathrm{b}}^{(1)}\right),($ bulk $)$ & $(2 \div 10) \times 10^{-3}$ & & & & \\
\hline$v_{l}\left(\mathbf{k}_{\mathrm{b}}^{(1)}\right),($ film $)$ & $(6 \div 25) \times 10^{-4}$ & & & & \\
\hline$v_{l}\left(\mathbf{k}_{\mathrm{b}}^{(2)}\right),($ film $)$ & $(1 \div 4) \times 10^{-3}$ & typ. $5 \times 10^{-4}$ & $2 \times 10^{-3} \rightarrow 4 \times 10^{-4}$ for $\theta_{i}=30^{\circ} \rightarrow 70^{\circ}$ & - & - \\
\hline$v_{t}\left(\mathbf{k}_{\mathrm{b}}^{(2)}\right),($ film $)$ & $(4 \div 15) \times 10^{-3}$ & & & & \\
\hline$v_{l}\left(\mathbf{k}_{\mathrm{b}}^{(3)}\right), v_{t}\left(\mathbf{k}_{\mathrm{b}}{ }^{(3)}\right)$, (bulk) & $(1 \div 9) \times 10^{-3}$ & typ. $5 \times 10^{-4}$ & 0 & $6 \times 10^{-4}$ & $\sim 2 \times 10^{-3}$ \\
\hline$v_{l}\left(\mathbf{k}_{\mathrm{b}}^{(4)}\right)$ & & & & & \\
\hline$v_{t}\left(\mathbf{k}_{\mathrm{b}}^{(4)}\right),($ bulk $)$ & $(1 \div 7) \times 10^{-3}$ & typ. $5 \times 10^{-4}$ & 0 & $2 \times 10^{-3}$ & 0 \\
\hline$v_{R}\left(\mathbf{k}_{\mathrm{s}}\right),($ bulk $)$ & $(7 \div 20) \times 10^{-3}$ & typ. $5 \times 10^{-4}$ & $2 \times 10^{-3} \div 4 \times 10^{-4}$ for $\theta_{i}=30^{\circ} \div 70^{\circ}$ & - & - \\
\hline$v_{R}\left(\mathbf{k}_{\mathrm{s}}\right),($ film $)$ & $(4 \div 25) \times 10^{-3}$ & & & & \\
\hline
\end{tabular}

Concerning frequency alone, it can also be noted that in the evaluation of the ratio $f_{1} / f_{2}$ of the frequencies of two doublets of the same spectrum the denominator of Eq. (4) cancels; the ratio is not affected by uncertainties due to calibration or to the FSR value (it can be measured without calibrating the spectrum). If furthermore the doublets are due to different modes probed in the same geometry, as the doublets in Figs. 3 and 4 connected with a circle marker, the ratio $f_{1} / f_{2}$ supplies a value of the ratio $v_{1} / v_{2}$ of the velocities of the two modes which is also insensitive to any uncertainty of the wavevector and to the broadening caused by the collection angle.

If repeated measurements are performed, weighted averages can be computed, the above estimates of the uncertainties being available for each doublet. In the assignment of the weights, only the values of $\sigma_{v^{\prime}}$, which characterize the lack of precision, must be considered. Therefore, for each spectrum and then for the average values, the uncertainties connected to each doublet are considered in successive steps. Firstly, the channel numbers of the peaks are considered, obtaining for each doublet the value (see Eqs. (4) and (7)) $n_{p}=n_{p}^{+}-n_{p}^{-}$, with its uncertainty $\sigma_{p}$. For doublets due to different modes probed in the same geometry, the ratio of the frequencies $f_{1} / f_{2}$ is immediately evaluated, with its uncertainty $\sigma_{f 1 / f 2}$, since it gives a value of the ratio $v_{1} / v_{2}$, which is insensitive to other uncertainties. The weighted average $\overline{f_{1} / f_{2}}$ is then computed.

Secondly, for each doublet, the velocity $v^{\prime}$ and its uncertainty $\sigma_{v^{\prime}}$ are obtained from the value of $f$ and the geometry. With weights determined by the $\sigma_{v^{\prime}}$ values, the average $\overline{v^{\prime}}$ is computed, with its uncertainty $\sigma_{\overline{v^{\prime}}}$. For the exchanged wavevectors independent from $n$, they coincide with $\bar{v}$ and $\sigma_{\bar{v}}$. Otherwise, $\bar{v}=\overline{v^{\prime}} / n, \sigma_{\bar{v}}^{\prime}=\sigma_{\overline{v^{\prime}}} / n$, and $\sigma_{\bar{v}}$, obtained as in Eq. (6), includes the lack of precision and the inaccuracy. Then, in the derivation of the elastic constants, the uncertainty $\sigma_{\rho}$ of the mass density must also be considered, such as $\sigma_{n}$, it affects accuracy but not precision.

Several spectra were recorded, to test different geometries and to assess the reproducibility of results. The results are summarized in the tables of Part E of the Supplementary material. ${ }^{1}$ For each of the velocities $v_{l}, v_{t}$, and $v_{R}$, and for each of the geometries, the tables report the number of measured doublets, the ranges of the values of velocity $v$ and of uncertainty $\sigma_{v}$, and the weighted averages $\bar{v}$ with the variances $\sigma_{\bar{v}}$ and $\sigma_{\bar{v}}^{\prime}$. Also for the ratios $v_{l} / v_{t}$, and (in Table E.II) $v_{R} / v_{t}$, the ranges of the values of $f_{1} / f_{2}$ and of $\sigma_{f 1 / f 2}$ are given, with the weighted average $\overline{v_{1} / v_{2}}$ and its variance $\sigma_{\overline{v_{1} / v_{2}}}$.

In Part E of the Supplementary material, it is argued that the effects of the finite light collection angle, briefly discussed in Part C of the Supplementary material, can be nonnegligible in some geometries. In particular, for the bulk specimen, it is noted that the average velocities measured in the same direct backscattering geometry (exchanged wavevector $\mathbf{k}_{\mathrm{b}}{ }^{(1)}$ ), but in different overall configurations, are in strict agreement, while those obtained in other geometries (exchanged wavevectors $\mathbf{k}_{\mathrm{b}}{ }^{(3)}$ and $\mathbf{k}_{\mathrm{b}}{ }^{(4)}$ ) differ from the first ones by up to above $2 \%$, several times the variances of the weighted averages. These discrepancies are in qualitative agreement with the discussion in Part C of the Supplementary material of the effects of the collection angle. Instead, when the ratio of velocities $v_{l} / v_{t}$ is measured by the $\mathrm{BL}$ and $\mathrm{BT}$ modes probed at a same wavevector, the ratios turn out to be in strict agreement for all the geometries. The two modes being probed at the same wavevector, any effect of inaccuracy or broadening of that wavevector is canceled in the ratio. On the other hand, for the film specimen, the $v_{l}$ values measured in the direct and indirect backscattering geometries (exchanged wavevectors $\mathbf{k}_{\mathbf{b}}{ }^{(1)}$ and $\mathbf{k}_{\mathbf{b}}{ }^{(2)}$ ) are in strict agreement; this is probably connected to the detailed computation of the effects of the finite collection angle for these geometries, ${ }^{29,43}$ that was exploited in the fitting procedure. Since a similar detailed analysis of the collection angle for the $\mathbf{k}_{\mathrm{b}}{ }^{(3)}$ and $\mathbf{k}_{\mathrm{b}}{ }^{(4)}$ exchanged wavevectors is not yet available, in this work the values of the ratio $f_{l} l f_{t}$ measured in these scattering geometries are considered on the same ground of those obtained in other geometries, while the absolute values of velocities are not further considered. 


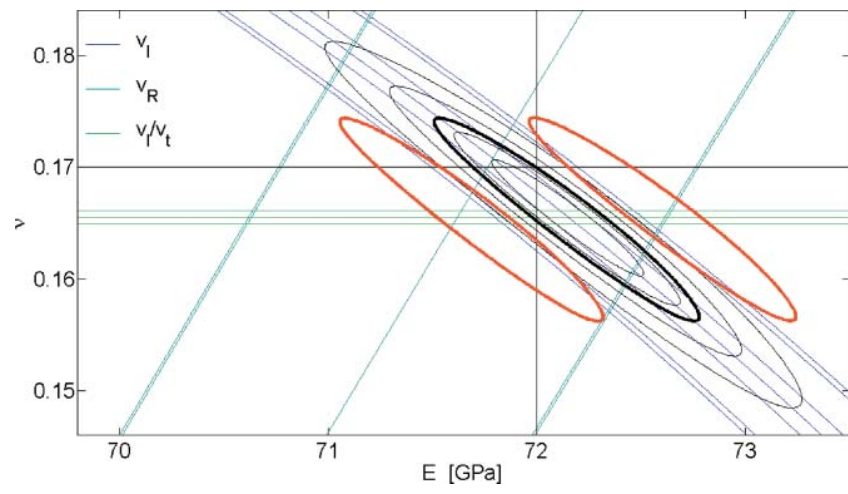

FIG. 5. (Color online) The two analysis procedures for one of the subsets of 15 doublets from the bulk sample. For the measured value $\overline{v_{l}}$, the central curve corresponds to the value $C_{11}=\rho_{b}{\overline{v_{l}}}^{2}$, while couples of curves delimit three nested bands, which correspond to the three terms of Eq. (D.6). The inner band is determined by the precision of $\overline{v_{l}}$, the next band includes the inaccuracy due to $n_{b}$, the outer band also includes the inaccuracy due to $\rho_{b}$. Analogous curves are drawn for $\overline{v_{R}}$ (for which the third band coincides with the second) and $\overline{v_{l} / v_{t}}$ (for which the two outer bands coincide with the inner one). The approximately elliptical curves are the isolevel curves of the normalized estimator $S^{\prime}$, at the $68 \%, 90 \%, 95 \%, 99 \%$, and $99.9 \%$ confidence level. The thicker curve is the $95 \%$ confidence region and the two translated replicas are the $95 \%$ confidence regions obtained by modified values of $n_{b}$ and $\rho_{b}$. The nominal values $E=72 \mathrm{GPa}, v=0.17$, supplied with the specimen to calibrate the indenters, are also shown.

\section{RESULTS AND DISCUSSION}

From the measured velocities, summarized in the tables of Part E of the Supplementary material, the elastic moduli can be derived in different ways. This step is basically independent from the technique by which the velocities are measured, either BS or others. It must be remembered that the stiffness of an isotropic solid is a two-dimensional quantity which can be represented in a two-dimensional "stiffness space." In Figs. 5 and 6, this space is represented by the $(E, v)$ couple, but, by a (non-linear) transformation of coordinates, it can be represented by any couple of independent moduli (see Eqs. (A.1)-(A.3)). Two procedures are considered here to derive the stiffness.

In the first one, the weighted averages $\bar{v}_{l}, \bar{v}_{t}, \bar{v}_{R}$, and $\overline{v_{l} / v_{t}}$ are computed; each of them puts a constraint on the position of the representative point in the "stiffness space," a condition such as $C_{11}=\rho{\overline{v_{l}}}^{2}$ identifies a curve in the $(E, v)$ plane or a line in the $\left(C_{11}, C_{44}\right)$ plane. All the constraints, which are consistent only to a finite degree, should be simultaneously considered.

The second procedure combines all the single measurement in the least squares estimator $S$, defined as the weighted sum over all the doublets; representing stiffness by the $(E, v)$ couple,

$$
\begin{aligned}
S(E, v)= & \sum_{i}\left(\frac{v_{l}(E, v)-v_{l, i}}{\sigma_{v_{l, i}}^{\prime}}\right)^{2}+\sum_{m}\left(\frac{v_{t}(E, v)-v_{t, m}}{\sigma_{v_{t, m}}^{\prime}}\right)^{2} \\
& +\sum_{k}\left(\frac{v_{R}(E, v)-v_{R, k}}{\sigma_{v_{R, k}}}\right)^{2} \\
& +\sum_{j}\left(\frac{v_{l} / v_{t}(E, v)-\left(v_{l} / v_{t}\right)_{j}}{\sigma_{\left(v_{l} / v_{t}\right)_{j}}}\right)^{2}
\end{aligned}
$$

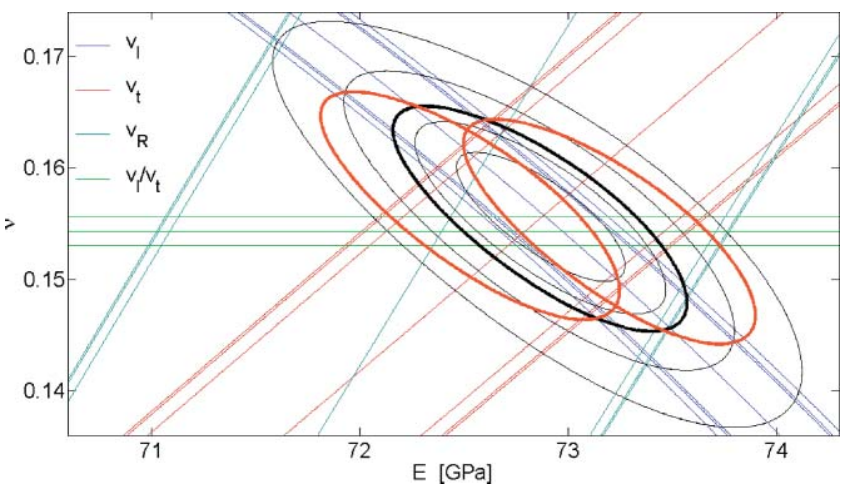

FIG. 6. (Color online) The two analysis procedures for one of the subsets of 15 doublets from the film sample. For the measured value $\overline{v_{l}}$ the central curve corresponds to the value $C_{11}=\rho_{f} \bar{v}^{2}$, then couples of curves delimit three nested bands, which correspond to the three terms of Eq. (D.8). The inner band is determined by the precision of $\overline{v_{l}}$, the next band includes the inaccuracy due to $n_{f}$ (also including the indirect effect by the mass density, see Part D of the Supplementary material in the supplementary material), the outer band also includes the inaccuracy due to $\rho_{b}$. Analogous curves are drawn for $\overline{v_{t}}, \overline{v_{R}}$, and $\overline{v_{l} / v_{t}}$. For $\overline{v_{l} / v_{t}}$, the two outer bands coincide with the inner one. The approximately elliptical curves are the isolevel curves of the normalized estimator $S^{\prime}$, at the $68 \%, 90 \%, 95 \%, 99 \%$, and $99.9 \%$ confidence level. The thicker curve is the $95 \%$ confidence region and the two translated and partially overlapped replicas are the $95 \%$ confidence regions obtained by modified values of $n_{f}$ and $\rho_{b}$.

where $v_{l, i}$ is the $i$ th value of $v_{l}$, of uncertainty $\sigma_{v_{l, i}}^{\prime}$, and analogous notations are adopted for $\left(v_{l} / v_{t}\right)_{j}, v_{R, k}$, and $v_{t, m}$. In both procedures, the weights are assigned to the single values, such as $v_{l, i}$, by the variances $\sigma_{v}^{\prime}$ which characterize precision alone.

Since BS measurements are time consuming, it is unlikely that as many spectra are recorded as those gathered in this work. To assess the precision and accuracy achievable by a reasonable number of spectra and the robustness of results, different measurement campaigns are simulated, for each of the two samples, by picking, among all the measured doublets, several subsets of results. For each of the two samples a first group is formed by 12 subsets of 8 doublets each, and a second group by 12 subsets of 15 doublets each. The subsets are picked at random, but selecting only those which minimizes the overlap, obtaining several subsets which, although not fully independent, have a minimal overlap. More details on the composition of the subsets are given in Part F of the Supplementary material. ${ }^{1}$ The "stiffness spaces" for one subset of 15 doublets from the bulk specimen and one from the film specimen are presented in Figs. 5 and 6.

For the bulk specimen, the first procedure works as follows. Each averaged velocity $\bar{v}$, such as, e.g., $\overline{v_{l}}$, is associated to the uncertainties $\sigma_{\bar{v}}^{\prime}$, which characterizes the finite precision, and $\sigma_{\bar{v}}$, which includes the effect of the possible inaccuracy of $n_{b}$. Accordingly, in the "stiffness space," the curve $C_{11}=\rho_{b}{\overline{v_{l}}}^{2}$ is drawn, with the three couples of curves $C_{11}$ $=\rho_{b}\left(\overline{v_{l}} \pm \sigma_{\overline{v_{l}}}^{\prime}\right)^{2}, \quad C_{11}=\rho_{b}\left(\overline{v_{l}} \pm \sigma_{\overline{v_{l}}}\right)^{2}, \quad$ and $\quad C_{11}=\rho_{b}{\overline{v_{l}}}^{2}$ $\pm \sigma_{C_{11}}$; the overall uncertainty $\sigma_{C}$ includes the uncertainty of $\rho_{b}$ and is given by Eq. (D.6). The three couples of curves correspond to the three terms of Eq. (D.6), and identify three nested bands, showing the effects of the finite precision, respectively, and the possible inaccuracies of $n_{b}$ and of $\rho_{b}$. In particular, $\sigma_{n} / n$ contributes, in some geometries, to $\sigma_{v} / v$ 
(Eq. (6)), and therefore, only through $\sigma_{v}$, to $\sigma_{C}$. Figure 5 presents these curves except for $C_{44}=\rho_{b}{\overline{v_{t}}}^{2}$ which, as discussed below, is not considered in the evaluation of the estimator.

For the film sample, the same procedure must take into account that the mass density $\rho_{f}$ is derived from $n_{f}$ and $\rho_{b}$ (see Sec. III). Therefore, an uncertainty $\sigma_{n, f}$ gives a twofold contribution to the overall uncertainty $\sigma_{C}$. By the first one, as in the bulk case, $\sigma_{n} / n$ contributes to $\sigma_{v} / v$, and through it to $\sigma_{C} / C$, only when the exchanged wavevector depends on $n$; by the second one it contributes to $\sigma_{C} / C$ indirectly, through the mass density $\rho_{f}$, for all the exchanged wavevectors (namely, by the terms in Eqs. (D.7) and (D.8) coming from the derivative (D.2.c)). Equations (D.7) and (D.8) also show that the direct effect through the velocity $v$, when present, partially compensates the indirect effect. Therefore, the exchanged wavevectors independent from $n$, in the bulk case imply a null sensitivity to $\sigma_{n} / n$ and in the film case imply instead a stronger sensitivity. Accordingly, in Fig. 6 the curve $C_{11}=\rho_{f}{\overline{v_{l}}}^{2}$ is drawn with three nested bands, which correspond here to the three terms of Eq. (D.8). While the first and last bands have the same meaning as in the bulk case; the intermediate one is not given by $C_{11}=\rho_{f}\left(\overline{v_{l}} \pm \sigma_{\overline{v_{l}}}\right)^{2}$, but by the combined indirect and (if present) direct effect of $\sigma_{n} / n$. The analogous curves are drawn, in Fig. 6, for $C_{44}=\rho_{f}{\overline{v_{t}}}^{2}$, for $\rho_{f}{\overline{v_{R}}}^{2}$ and for $C_{11} / C_{44}=\left(\overline{v_{l} / v_{t}}\right)^{2}$.

The subset whose results are shown in Fig. 5 gives a good precision for $\overline{v_{l}}$ : the inner band is narrow and the refractive index and mass density give substantial contributions. The precision for $\overline{v_{R}}$ is much lower, the lack of precision dominates the uncertainty, the mass density only adding a negligible contribution. The subset whose results are shown in Fig. 6 is among those that give a lower precision; accordingly; for all the velocities, the inner band is dominant, and the contributions from refractive index and mass density are minor. The various bands intersect in the same region, although not exactly at the same point, confirming the need of considering all the indications coming from $\bar{v}_{l}, \bar{v}_{t}, \bar{v}_{R}$, and $\overline{v_{l} / v_{t}}$. The smallness of the intersection region is however remarkable, denoting a high consistency of the results, in particular for the film sample.

The second procedure exploits the estimator $S(E, \nu)$ of Eq. (8). In its computation, only truly independent results must be considered. For the bulk specimen, as discussed above, the results obtained by the exchanged wavevectors $\mathbf{k}_{\mathbf{b}}{ }^{(3)}$ and $\mathbf{k}_{\mathbf{b}}{ }^{(4)}$ are considered only for the ratio $v_{l} / v_{t}$, not for the separate velocities. Three groups of results are then considered: firstly, the values of $v_{l}$ measured by the exchanged wavevector $\mathbf{k}_{\mathrm{b}}{ }^{(1)}$; each of them contributes by a term in the sum of $\left(v_{l}(E, v)-v_{l, i}\right)$. Secondly, the values of $v_{t}$, by all the geometries which measure it, each of them can contribute by either a term in the sum of $\left(v_{t}(E, \nu)-v_{t, m}\right)$ or by a term in the sum of $\left(v_{l} / v_{t}(E, v)\right.$ - $\left.\left(v_{l} / v_{t}\right)_{j}\right)$, but not both. The ratio $v_{l} / v_{t}$ is preferred because it is insensitive to the uncertainties due to the exchanged wavevector. The third group is formed by the values of $v_{R}$. For the film specimen, the velocity $v_{l}$ is measured by both the wavevectors $\mathbf{k}_{\mathrm{b}}{ }^{(1)}$ and $\mathbf{k}_{\mathrm{b}}{ }^{(2)}$, and $v_{t}$ by the wavevector $\mathbf{k}_{\mathbf{b}}{ }^{(2)}$. Four independent groups are consid- ered, again chosen because they are the more precise ones, $v_{l}$ by $\mathbf{k}_{\mathrm{b}}{ }^{(1)}$ alone, $v_{t}$ by $\mathbf{k}_{\mathrm{b}}{ }^{(2)}, v_{l} / v_{t}$ by $\mathbf{k}_{\mathrm{b}}{ }^{(2)}$, and the values of $v_{R}$.

A detailed statistical analysis of the results would require techniques of analysis of variance (ANOVA), which are beyond the scope of this work. A more heuristic analysis was instead performed, exploiting the result of estimation theory which identifies the confidence regions for the estimated parameters by the isolevel curves of the normalized estimator, ${ }^{44}$

$$
S^{\prime}(E, v)=\frac{S(E, v)-S_{\min }}{S_{\min }} .
$$

The normalized estimator $S^{\prime}(E, v)$ is computed at the nodes of a discrete mesh, which is refined until stability upon a further refinement is reached. For all, the subsets the estimator $S^{\prime}$ has the shape of a well defined well, of approximately elliptical section.

For the estimation of two parameters from eight measurements, the confidence regions at $68 \%, 90 \%, 95 \%, 99 \%$, and $99.9 \%$ confidence levels are given by the isolevel curves of $S^{\prime}$ at the values of $0.462,1.154,1.714,3.641$, and 8.999 , respectively; for the estimation of 2 parameters from 15 measurements the values are $0.1916,0.4251,0.5854,1.031$, and 1.894, respectively. ${ }^{44}$ The confidence regions turn out to be the most robust result of this analysis. The numerical "noise," connected to the discrete mesh over which $S^{\prime}$ is computed, is evaluated by varying the mesh resolution or by slightly shifting it. With the adopted mesh, the boundaries of the confidence regions turn out to always shift by less than $0.08 \%$, and in most cases by less than $0.04 \%$. The position of the minimum of the estimator $S(E, v)$ turns out to be more sensitive, probably due to the smallness of gradients in the neighborhood of the minimum.

The final results are, therefore, based on the confidence regions; from each of the subsets, the best estimates of $E$ and $v$ are picked as the midpoint between the lower and upper limits of the $95 \%$ confidence region, and the estimated uncertainty is taken as the semi-amplitude of this interval. Representing the "stiffness space" by other couples of moduli, such as $(B, G)$ or $\left(C_{11}, C_{44}\right)$, the estimates are found for all of them. It has been checked that the results for any modulus do not depend on the modulus to which it is coupled. An overview of these results is given in Part F of the Supplementary material. The estimator $S$, defined by Eq. (8), characterizes precision alone; as noted above, the subset of Fig. 5 gives a good precision and shows relatively narrow confidence regions, while the subset of Fig. 6, which gives a lower precision, presents wider confidence regions.

The values of the moduli reported in Part F of the Supplementary material are obtained with the central values of the refractive indexes and mass densities (see Sec. III): $\left(n_{b}\right.$ $=1.467$ and $\left.\rho_{b}=2200 \mathrm{~kg} \mathrm{~m}^{-3}\right)$ and $\left(n_{f}=1.488\right.$ and $\rho_{f}$ $=2284 \mathrm{~kg} \mathrm{~m}^{-3}$ ). The possible inaccuracies of these values are discussed in Sec. III and in Part D of the Supplementary material, and their effects are shown by the above mentioned bands in the "stiffness space." A more quantitative assessment is now performed, following the two analysis procedures to 
evaluate the consequences of small variations of these parameters alone.

For the bulk specimen the primary, uncorrelated, uncertainties are those of $\rho_{b}$ and $n_{b}$; their effects are evaluated by Eq. (D.5). For this sample, each of the considered subsets of results includes values of $v_{l}$ from $\mathbf{k}_{\mathrm{b}}{ }^{(1)}$, which are very precise and depend on $n$, values of $v_{R}$ from $\mathbf{k}_{\mathrm{s}}$, which are significantly less precise but do not depend on $n$, and values of $v_{l} / v_{t}$ which are precise and independent from $\rho$ and $n$. Equation (D.5) shows that the largest variations $\mathrm{d} C$ are due to $v_{l}$ from $\mathbf{k}_{\mathrm{b}}{ }^{(1)}$, when $\mathrm{d} \rho_{\mathrm{b}}$ and $\mathrm{d} n_{\mathrm{b}}$ have opposite signs: the variations (see Sec. III) $\left(\mathrm{d} \rho_{b} / \rho_{\mathrm{b}}, \mathrm{d} n_{b} / n_{b}\right)= \pm(+0.23 \%,-0.2 \%$,) imply $\mathrm{d} C / C= \pm 0.63 \%$. For $v_{R}$ from $\mathbf{k}_{\mathrm{s}}$, independent from $n_{b}$, we expect instead only the variation $\mathrm{d} C / C= \pm 0.23 \%$ due to $\mathrm{d} \rho_{b}$, and for $v_{l} / v_{t}$ no variation.

For the film specimen, the value of $\rho_{f}$ being derived from $\rho_{b}, n_{b}$, and $n_{f}$ (Eq. (D.1)), the primary, uncorrelated, uncertainties are those of these three parameters; their effects are evaluated by Eq. (D.7) ignoring, as discussed in Part D of the Supplementary material, the variations $\mathrm{d} n_{b}$. For this sample, each of the considered subsets includes quite precise values of $v_{l}$ from $\mathbf{k}_{\mathbf{b}}{ }^{(1)}$. Since this wavevector depends on $n_{f}$; the effect of $\mathrm{d} n_{f}$ is partially compensated, as already noted and shown by Eq. (D.7). The values from the other three groups, namely, $v_{t}$ from $\mathbf{k}_{\mathrm{b}}{ }^{(2)}, v_{l} / v_{t}$ from $\mathbf{k}_{\mathrm{b}}{ }^{(2)}$, and $v_{R}$ from $\mathbf{k}_{\mathrm{s}}$, have comparable relative precision, lower than that of $v_{l}$ from $\mathbf{k}_{\mathrm{b}}{ }^{(1)}$. For these groups, the wavevector does not depend on $n_{f}$, and the indirect effect of $\mathrm{d} n_{f}$ remains not compensated. In all cases, we expect larger variations for $\mathrm{d} \rho_{b}$ and $\mathrm{d} n_{f}$ of the same sign. For variations $\left(\mathrm{d} \rho_{b} / \rho_{b}, \mathrm{~d} n_{f} / n_{f}\right)= \pm(+0.23 \%,+0.2 \%$, , from Eq. (D.7), we have for $v_{l}$ from $\mathbf{k}_{\mathrm{b}}{ }^{(1)}$, the effect of $\mathrm{d} n_{f}$ being partially compensated, $\mathrm{d} C / C= \pm 0.35 \%$; for $v_{t}$ from $\mathbf{k}_{\mathrm{b}}{ }^{(2)}$ and $v_{R}$ from $\mathbf{k}_{\mathrm{s}}$, the effect of $\mathrm{d} n_{f}$ being not compensated, $\mathrm{d} C / C$ $= \pm 0.75 \%$; for $v_{l} / v_{t}$, independent from $\rho$ and $n, \mathrm{~d} C / C=0$.

An overall assessment is better performed by the second analysis procedure, re-computing the confidence regions with modified values of the mass density and refractive index. The computation is performed for the subsets of 15 doublets each, with the combinations of possible underestimations and overestimations which, according to the above evaluation, cause the most severe variations of the final results. For the bulk specimen, they are $\left(\mathrm{d} \rho_{b} / \rho_{b}, \mathrm{~d} n_{b} / n_{b}\right)= \pm(+0.23 \%,-0.2 \%$, ), meaning ( $\rho_{b}=2205, n_{b}=1.464 \mathrm{~kg} \mathrm{~m}^{-3}$, high stiffness) and $\left(\rho_{b}=2195, n_{b}=1.470 \mathrm{~kg} \mathrm{~m}^{-3}\right.$, low stiffness). For the film specimen, they are $\left(\mathrm{d} \rho_{b} / \rho_{b}, \mathrm{~d} n_{f} / n_{f}\right)= \pm(0.23 \%, 0.2 \%$, $)$ which imply (see Sec. III) $\mathrm{d} \rho_{f} / \rho_{f}= \pm 0.75 \%$, and lead to $\left(\rho_{f}\right.$ $=2301, n_{f}=1.491 \mathrm{~kg} \mathrm{~m}^{-3}$, high stiffness $)$ and ( $\rho_{f}=2267, n_{f}$ $=1.485 \mathrm{~kg} \mathrm{~m}^{-3}$, low stiffness). Figures 5 and 6 show the recomputed confidence regions, which are shifted without significant modifications of shape and size.

By a procedure analogous to that exploited above to identify the best estimates, the estimates which include the possible inaccuracies are taken as the midpoint between the lowest and highest limits of the ensemble of $95 \%$ confidence regions, i.e., the center of the rectangle, with sides parallel to the axes, circumscribed to all the shifted confidence regions; the semiamplitude is taken as the estimated uncertainty. Comparing these new estimates with those obtained with the nominal values of parameters, the shifts turn out to be not larger than the numerical noise, the uncertainties being obviously significantly wider. The main results are summarized in Table II which, for the narrowest and the widest of the "nominal" confidence regions, and for all the moduli, compares the uncertainties due to precision alone (i.e., those reported in Table F.I) with those which also consider the inaccuracy. The table also reports the relative difference between the highest and the lowest of the estimates given by the 12 subsets, and a "best estimate" for each parameter, computed as the weighted average among the results supplied by the 12 subsets.

It must be remembered that a non-negligible fraction of the uncertainty comes from the possible inaccuracy of the mass density values and that this contribution affects all the techniques based on vibrations in the same way, since they all need the value of inertia to derive the stiffness. If a very precise value of mass density is available, the final uncertainty is reduced at a level, which remains determined by the other sources of uncertainty.

The results found in this work can be compared with the nominal calibration values $E=72 \mathrm{GPa}$ and $v=0.18$ supplied for the bulk specimen and shown in Fig. 5, and with the values for bulky vitreous silica measured by ultrasonic techniques: ${ }^{45,46} v_{l}=5970 \mathrm{~m} / \mathrm{s}, v_{t}=3760 \mathrm{~m} / \mathrm{s}, E=72.9 \mathrm{GPa}$, $\mathrm{G}=31.2 \mathrm{GPa}, B=36.8 \mathrm{GPa}, v=0.182$. They can also be compared with the velocities measured at room temperature by a BS setup which reaches very high temperatures: ${ }^{47} v_{l}$ $=5953 \mathrm{~m} / \mathrm{s}$ and $v_{t}=3743$, with uncertainties estimated (probably in a conservative way) at 300 and $200 \mathrm{~m} / \mathrm{s}$, respectively. A value of mass density is not quoted, and the value $B=36.9 \mathrm{GPa}$ is found. Data are also available from several suppliers of amorphous silica for technological applications; the properties declared by several suppliers lie within the intervals: $E=72-74 \mathrm{GPa}, \mathrm{G}=30.6-32 \mathrm{GPa}, B=35.4-37.4$ $\mathrm{GPa}$ (with one supplier declaring $42 \mathrm{GPa}$ ), $v=0.17-0.18$, and refractive index close to 1.462 for wavelengths close to $500 \mathrm{~nm}$.

A similar thermally grown silica film was the object of earlier measurements, presented with a much simpler data analysis. ${ }^{48}$ The procedure presented here achieves not only a substantial reduction of the uncertainties; but also higher values of all the elastic moduli by around 5\%. This discrepancy, much larger than the presently estimated uncertainties, is easily tracked to the mass density value $\rho_{f}$. In the absence of specific measurements, the value $\rho_{f}=2200 \mathrm{~kg} / \mathrm{m}^{3}$ was exploited in the former analysis, while the ellipsometric measurements performed here lead to the value $\rho_{f}=2284 \mathrm{~kg} / \mathrm{m}^{3}$. Once this difference is taken into account, the difference between former and present results is reduced to about $1 \%$, well within the uncertainty estimated for the former results. This interpretation is confirmed by the values of Poisson's ratio, which is insensitive to $\rho_{f}$; although affected by a larger uncertainty, the former value differs from the present one by only $2 \%$. This comparison confirms the crucial role of mass density, which is shared by all the techniques, which exploit oscillations. The present results can also be compared to the values obtained in Ref. 10 for a $600 \mathrm{~nm}$ thick $\mathrm{SiO}_{2}$ film deposited on silicon by plasma enhanced chemical vapor deposition. Adopting a mass density of $2180 \mathrm{~kg} / \mathrm{m}^{3}$, of unspecified origin, the values $E=71.6 \mathrm{GPa}, v=0.16$ are found; uncertainties are not 
TABLE II. For the bulk and the film samples, for the moduli $E, B, G$, and $C_{11}$ and for Poisson's ratio $v$, for the values obtained from the 12 subsets of 15 doublets each: the values associated to the narrowest and the widest uncertainty intervals; the uncertainties due to finite precision alone (these are the same values of Table F.I), and those which also include the possible inaccuracies due to $n$ and $\rho$; the relative difference between the highest and the lowest estimates from the 12 subsets, the best estimate, given by the weighted average of the values given by the 12 subsets.

\begin{tabular}{|c|c|c|c|c|c|c|c|c|c|}
\hline \multirow[b]{2}{*}{ Sample } & \multirow[b]{2}{*}{ Quantity } & \multicolumn{3}{|c|}{ Narrow } & \multicolumn{3}{|c|}{ Wide } & \multirow[b]{2}{*}{ hi/lo $\Delta \%$} & \multirow[b]{2}{*}{ Best } \\
\hline & & Value & Precis. $\pm \%$ & Accur. $\pm \%$ & Value & Precis. $\pm \%$ & Accur. $\pm \%$ & & \\
\hline \multirow[t]{5}{*}{ Bulk } & $E[\mathrm{GPa}]$ & 72.25 & 0.5 & 1.1 & 72.07 & 2.1 & 2.8 & 0.3 & 72.29 \\
\hline & $v$ & 0.1678 & 2.9 & 3.0 & 0.1662 & 13.5 & 13.6 & 1.5 & 0.1659 \\
\hline & $B$ [GPa $]$ & 36.26 & 1.0 & 1.7 & 36.10 & 4.6 & 5.3 & 0.9 & 36.16 \\
\hline & $G=C_{44}[\mathrm{GPa}]$ & 30.94 & 0.9 & 1.5 & 30.93 & 4.0 & 4.7 & 0.4 & 30.96 \\
\hline & $C_{11}[\mathrm{GPa}]$ & 77.51 & 0.1 & 0.8 & 77.34 & 0.3 & 0.9 & 0.4 & 77.44 \\
\hline \multirow[t]{5}{*}{ Film } & $E[\mathrm{GPa}]$ & 72.75 & 0.6 & 1.1 & 72.64 & 1.1 & 1.6 & 0.7 & 72.73 \\
\hline & $v$ & 0.1535 & 4.6 & 5.7 & 0.1599 & 7.4 & 8.5 & 6.1 & 0.1574 \\
\hline & $B$ [GPa $]$ & 35.00 & 1.5 & 1.5 & 35.63 & 2.5 & 2.5 & 2.7 & 35.43 \\
\hline & $G=C_{44}[\mathrm{GPa}]$ & 31.54 & 1.2 & 1.8 & 31.32 & 2.0 & 2.7 & 1.2 & 31.42 \\
\hline & $C_{11}[\mathrm{GPa}]$ & 77.05 & 0.3 & 0.7 & 77.31 & 0.6 & 1.0 & 0.8 & 77.30 \\
\hline
\end{tabular}

quoted, but due to the needed extrapolation to null thickness of the aluminum interaction layer, they are probably larger than those found here.

\section{CONCLUSIONS}

Brillouin spectroscopy can provide a full characterization of the elastic behaviour of solids. The whole measurement process, from the optical setup to the derivation of the elastic moduli, has been reviewed identifying the various sources of uncertainty. A detailed procedure to assess the effect of each of them is developed, discriminating the effects on precision from those on accuracy. The assessment has been performed for a model material, silica, in bulk form and as a thermally grown film. Combining ellipsometry with BS, a fully optical characterization route is obtained. The following results are found, for the characterization of isotropic media.

The raw outcome of BS measurements is the frequency of spectral doublets. The frequency calibration is affected by a typical uncertainty of a few parts in 10000 and a doublet frequency is identified with a precision which depends on the peak intensity; from a few parts in 10000 for the most intense doublets, up to some parts in a hundred for the weaker ones. By repeated measurements, the uncertainty of the weighted average can be lowered to the level of one part in a thousand. This is the intrinsic precision of frequencies measured by BS.

The velocity of the acoustic modes is obtained from the doublet frequency and the exchanged wavevector, which depends on the scattering geometry and/or the refractive index. The doublet frequencies and the geometry are affected by random errors, which limit the precision, but do not affect accuracy. Errors due to geometrical imperfections can be lowered to the one part in a thousand level. Ellipsometry provides the refractive index with an uncertainty of few parts in a thousand, which does not affect precision, but limits the accuracy of velocity.

As in all the methods based on vibrations, elastic moduli can be derived from the measured velocity exploiting an independently measured value of the mass density; the results are subjected to the possible inaccuracies of density, which, par- ticularly in the case of films, is not always known to be better than one part in a thousand.

The precision of the elastic moduli and Poisson's ratio is well evaluated by the confidence regions, identified by a least square estimator. The effect on the confidence regions measures the accuracy limiting effect of the auxiliary parameters (refractive index and mass density).

By a comprehensive analysis of the whole measurement process, this work qualifies the performance of the measurement technique based on Brillouin spectroscopy. It is shown that for the measurement of the stiffness of an isotropic transparent medium, including a micrometric film, Brillouin spectroscopy, exploited in conjunction with ellipsometry, can achieve precisions and accuracies of the order of $1 \%$, which are difficultly obtainable by other techniques. Brillouin spectroscopy, a contactless light scattering technique, which does not need a specific sample preparation, is thus qualified as a high precision technique.

${ }^{1}$ See supplementary material at http://dx.doi.org/10.1063/1.3585980 for Parts A-F.

${ }^{2}$ J. Hay, J. Mater. Res. 24, 667 (2009); Z. Wei, G. Zhang, H. Chen, J. Luo, R. Liu, and S. Guo, ibid. 24, 801 (2009); M. Sakai, ibid. 24, 831 (2009).

${ }^{3}$ B. A. Walmsley, A. J. Keating, Y. Liu, Z. Hu, M. B. Bush, J. M. Dell, and L. Faraone, J. Appl. Phys. 102, 103517 (2007).

${ }^{4}$ N. Nakamura, T. Nakashima, H. Ogi, M. Hirao, and M. Nishiyama, J. Appl. Phys. 107, 103541 (2010).

${ }^{5}$ N. Nakamura, T. Nakashima, S. Oura, H. Ogi, and H. Hirao, Ultrasonics 50, 150 (2010).

${ }^{6}$ D. Schneider, Th. Witke, Th. Schwarz, B. Schöneich, and B. Schultrich, Surf. Coat. Technol. 126, 136 (2000).

${ }^{7}$ J. Bryner, D. M. Profunser, J. Vollmann, E. Mueller, and J. Dual, Ultrasonics 44, e1269 (2006).

${ }^{8}$ H. Ogi, M. Fujii, N. Nakamura, and T. Yasui, Phys. Rev. Lett. 98, 195503 (2007).

${ }^{9}$ H. Ogi, M. Fujii, N. Nakamura, T. Shagawa, and M. Hirao, Appl. Phys. Lett. 90, 191906 (2007).

${ }^{10}$ P. A. Mante, J. F. Robillard, and A. Devos, Appl. Phys. Lett. 93, 071900 (2008).

${ }^{11}$ J. R. Sandercock, in Light Scattering in Solids III, edited by M. Cardona and G. Güntherodt (Springer, Berlin, 1982).

${ }^{12}$ M. G. Beghi, A. G. Every, and P. V. Zinin, in Ultrasonic Nondestructive Evaluation, edited by T. Kundu (CRC Press, Boca Raton, FL, 2004). 
${ }^{13}$ P. Mutti, C. E. Bottani, G. Ghislotti, M. Beghi, G. A. D. Briggs, and J. R. Sandercock, in Advances in Acoustic Microscopy, edited by A. Briggs (Plenum, New York, 1995).

${ }^{14}$ M. Grimsditch, in Handbook of Elastic Properties of Solids, Liquids, and Gases, edited by M. Levy, H. E. Bass, and R. R. Stern (Academic, New York, 2001).

${ }^{15}$ J. D. Comins, in Handbook of Elastic Properties of Solids, Liquids, and Gases, edited by M. Levy, H. E. Bass, and R. R. Stern (Academic, New York, 2001).

${ }^{16}$ A. G. Every, Meas. Sci. Technol. 13, R21 (2002).

${ }^{17}$ M. G. Beghi, A. C. Ferrari, K. B. K. Teo, J. Robertson, C. E. Bottani, A. Libassi, and B. K. Tanner, Appl. Phys. Lett. 81, 3804 (2002).

${ }^{18}$ R. D. Hartschuh, A. Kisliuk, V. Novikov, A. P. Sokolov, P. R. Heyliger, C. M. Flannery, W. L. Johnson, C. L. Soles, and W. L. Wu, Appl. Phys. Lett. 87, 173132 (2005).

${ }^{19}$ S. Sinogeikin, J. Bass, V. Prakapenka, D. Lakshtanov, G. Shen, C. SanchezValle, and M. Rivers, Rev. Sci. Instrum. 77, 103905 (2006).

${ }^{20}$ W. Cheng, R. Sainidou, P. Burgardt, N. Stefanou, A. Kiyanova, M. Efremov, G. Fytas, and P. F. Nealey, Macromolecules 40, 7283 (2007).

${ }^{21}$ T. Still, W. Cheng, M. Retsch, U. Jonas, and G. Fytas, J. Phys. Condens. Matter 20, 404203 (2008).

${ }^{22}$ N. Gomopoulos, W. Cheng, M. Efremov, P. F. Nealey, and G. Fytas, Macromolecules 42, 7164 (2009).

${ }^{23}$ K. L. Kearns, T. Still, G. Fytas, and M. D. Ediger, Adv. Mater. 22, 39 (2010).

${ }^{24}$ N. Lou, J. Groenen, G. Benassayag, and A. Zwick, Appl. Phys. Lett. 97, 141908 (2010).

${ }^{25}$ M. Murakami and J. D. Bass, Phys. Rev. Lett. 104, 025504 (2010).

${ }^{26}$ B. Chen, A. E. Gleason, J. Y. Yan, K. J. Koski, S. Clark, and R. Jeanloz, Phys. Rev. B 81, 144110 (2010).

${ }^{27}$ M. Kawashima, Y. Matsuda, S. Aramomi, and S. Kojima, Jpn. J. Appl. Phys. 49, 07HB02 (2010).

${ }^{28}$ J. R. Sandercock, Solid State Commun. 26, 547 (1978).

${ }^{29}$ P. R. Stoddart, J. C. Crowhurst, and J. D. Comins, J. Opt. Soc. Am. B 15, 2481 (1998).
${ }^{30}$ A. G. Every, in Handbook of Elastic Properties of Solids, Liquids, and Gases, edited by M. Levy, H. E. Bass, and R. R. Stern (Academic, New York, 2001).

${ }^{31}$ Process THELMA by ST Microelectronics, for the production of micro electro-mechanical systems (MEMS).

${ }^{32}$ J. M. Vaughan, The Fabry-Perot Interferometer (Adam Hilger, Bristol, 1989).

${ }^{33}$ S. M. Lindsay, M. W. Anderson, and J. R. Sandercock, Rev. Sci. Instrum. 52, 1478 (1981).

${ }^{34}$ J. R. Sandercock, TFP-1 Interferometer Operator Manual (JRS Scientific Instruments, Zwillikon, CH, 2001).

${ }^{35}$ X. Zhang, R. Sooryakumar, A. G. Every, and M. H. Manghnani, Phys. Rev. B 64, 081402 (2001).

${ }^{36}$ R. M. A. Azzam and N. M. Bashara, Ellipsometry and Polarized Light (Elsevier, Amsterdam, 1992).

${ }^{37}$ B. M. Ayupov, V. A. Grtsenko, H. Wong, and C. W. Kim, J. Electrochem. Soc. 153, F277 (2006).

${ }^{38}$ D. Chandler-Horowitz and G. A. Candela, J. Phys. Colloques 44, C10-23 (1983).

${ }^{39}$ C. M. Herzinger, B. Johs, W. A. McGahan, J. A. Woollam, and W. Paulson, J. Appl. Phys. 83, 3323 (1998).

${ }^{40}$ E. D. Palik, Handbook of Optical Constants of Solids (Academic, New York, 1985).

${ }^{41}$ N. W. Ashcroft and N. D. Mermin, Solid State Physics (ThomsonBrooks/Cole, Pacific Grove, CA, 2003).

${ }^{42}$ R. Vogelgesang, A. K. Ramdas, S. Rodriguez, M. Grimsditch, and T. R. Anthony, Phys. Rev. B 54, 3989 (1996).

${ }^{43}$ M. G. Beghi, "Light collection angle effects in Brillouin spectroscopy" (unpublished).

${ }^{44}$ G. A. F. Seber and C. J. Wild, Nonlinear Regression (Wiley-Interscience, Hoboken, NJ, 2003).

${ }^{45}$ H. J. McSkimin, J. Acoust. Soc. Am. 31, 287 (1959).

${ }^{46}$ O. L. Anderson, in Physical Acoustics, edited by W. P. Mason (Academic, New York 1965), Vol. III B.

${ }^{47}$ A. Polian, D. Vo-Thanh, and P. Richet, Europhys. Lett. 57, 375 (2002).

${ }^{48}$ G. Signoriello and M. G. Beghi, Sens. Lett. 6, 130 (2008). 